A

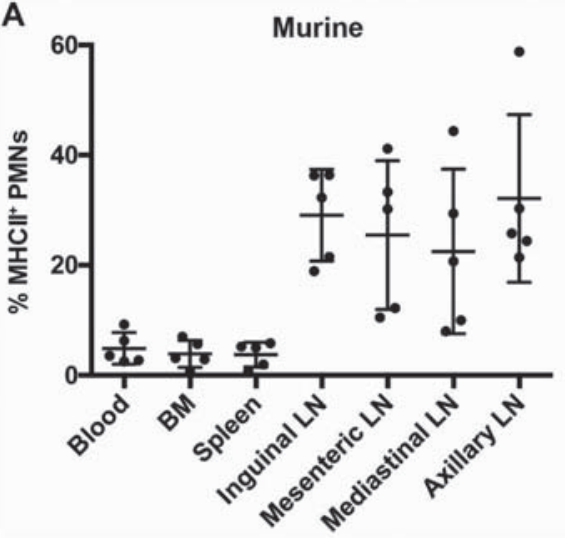

B

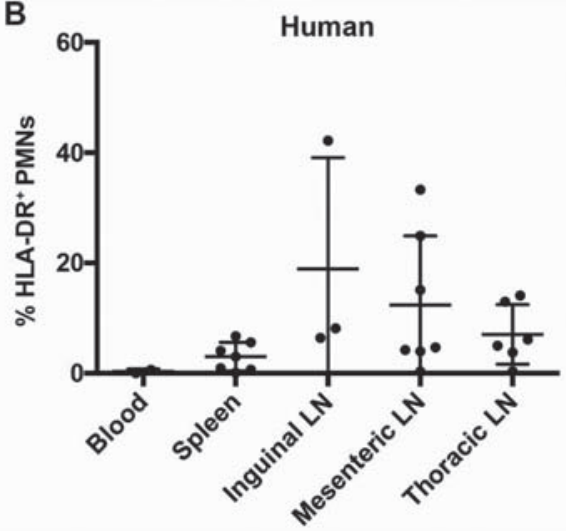

Abstract S19 Figure 1 Surface MHCII expression of tissue-resident neutrophils in (A) mice and (B) humans. Lines represent mean \pm S.D.

nodes following infectious challenges, the role of tissue-resident neutrophils in physiological settings is less clear. We hypothesise that neutrophils are present within lymph nodes and can influence adaptive immunity under physiological conditions.

Methods Lymph nodes from unchallenged C57BL/6 and LysM-GFP mice were harvested; single cell suspensions were generated for flow cytometric analysis, and frozen sections stained for confocal microscopy. Two-photon intravital imaging of popliteal lymph nodes was performed to examine neutrophil dynamic behaviour in vivo. Human lymph nodes were harvested from organ donors and analysed by flow cytometry and mass cytometry.

Results Neutrophils were present in lymph nodes in mice without prior inflammatory or infectious challenge. Whilst some neutrophils were within blood vessels $(11 \%$ in inguinal lymph node, $10 \%$ in popliteal lymph node, $12 \%$ in mesenteric lymph node) or lymphatic vessels $(15 \%$ in inguinal lymph node, $21 \%$ in popliteal lymph node, $18 \%$ in mesenteric lymph node), the majority were located in lymph node tissues. Lymph node neutrophils showed higher surface expression of major histocompatibility complex II (MHCII) compared to blood, bone marrow and splenic neutrophils (figure 1A). In vivo, neutrophils were capable of immune complex uptake, and their dynamic behaviour differed according to their location within the lymph node. Neutrophils were also present in human lymph nodes, and expressed surface MHCII (figure 1B). Immune cell profiles of matched lymph nodes and spleen were compared using mass cytometry. Isolated human blood neutrophils upregulated surface MHCII upon ex vivo immune complex stimulation.

Conclusion We have demonstrated the presence of tissue-resident neutrophils within murine and human lymph nodes, and their capacity to express MHCII, potentially influencing the adaptive immune response via antigen presentation.

\section{S20 IMPACT OF AZITHROMYCIN ON THE POST-LUNG TRANSPLANT MICROBIOTA}

${ }^{1} \mathrm{C}$ Spence ${ }^{*},{ }^{2} S$ Verleden ${ }^{*},{ }^{1} \mathrm{G}$ Einarsson, ${ }^{2} \mathrm{~J}$ Yserbyt, ${ }^{1} \mathrm{AJ}$ Lee, ${ }^{2} \mathrm{~A}$ Van Herck, ${ }^{1} \mathrm{E}$ Johnston, ${ }^{1} \mathrm{JS}$ Elborn, ${ }^{2} \mathrm{GM}$ Verleden, ${ }^{1} \mathrm{DF}$ Gilpin, ${ }^{2} \mathrm{~B}$ Vanaudenaerde, ${ }^{1} \mathrm{MM}$ Tunney*, ${ }^{2} \mathrm{R}$ Vos ${ }^{*}{ }^{1} \mathrm{Halo}$ Research Group, Queen's University Belfast, Belfast, UK; ${ }^{2}$ Lung Transplant Unit, University of Leuven, Leuven, Belgium; *Authors contributed equally to this work
Introduction and Objectives Chronic Lung Allograft Dysfunction (CLAD) is a major limiting factor to survival post-lung transplant (LTx), restricting 5 year survival to approximately $55 \%$. The mechanism by which CLAD and its sub-types occur are not fully understood and changes in the microbiota may play a role in the development of this condition. Moreover, azithromycin prolongs CLAD-free post-transplantation. This study aims to determine the effect of azithromycin over time on the airway microbiota post-LTx and how the microbiota changes with the development of CLAD.

Methods As part of a double-blind RCT in UZ Gasthuisberg, Leuven, Belgium, ${ }^{1}$ patients undergoing LTx were previously randomised to receive either azithromycin ( $\mathrm{n}=43 ; 250 \mathrm{mg}$ three times per week) or placebo $(n=40)$ treatment following discharge post-transplant. Regular routine bronchoscopy was carried out on all patients and bronchoalveolar lavage (BAL) samples from discharge, 12, 24 months and at diagnosis of suspected rejection were processed for microbiota analysis using 16S Illumina sequencing and 16S quantitative PCR.

Results To date, 42 azithromycin treated ( $\mathrm{n}=17$ patients) and $52(\mathrm{n}=22$ patients) placebo samples have been analysed. Microbiota diversity was significantly higher $(p=0.0467)$ in the azithromycin group compared to placebo. Furthermore, a trend for reduced dominance by Pseudomonas, with re-emergence of taxa considered to constitute a 'healthy' microbiota (e.g., Prevotella, Veillonella, Streptococcus) was observed. There were no significant differences in $16 \mathrm{~S}$ copies per $\mathrm{mL}$ BAL between the two groups. Eight samples $(n=5$ azithromyin, $n=3$ placebo) at suspected rejection have also been analysed. The azithromycin group exhibited low relative abundances of Pseudomonas (mean 7.7\%), while the placebo group showed dominance by this taxa (mean $84.87 \%$ ).

Conclusions Restoration of a diverse microbiota, while preventing dominance by Pseudomonas, may be a factor contributing towards the prophylactic effects of azithromycin observed in LTx patients ${ }^{1}$. Further analysis of microbiota data alongside clinical data e.g., development of CLAD, CLAD-free survival time, etc. is ongoing.

\section{REFERENCE}

1. Vos R, Vanaudenaerde BM, Verleden SE, et al. A randomised controlled trial of azithromycin to prevent chronic rejection after lung transplantation. European Respiratory Journal 2011;37:164-172. 


\section{Pleural effusions: diagnosis and prognosis}

\section{S21 A PROSPECTIVE STUDY USING SERUM MESOTHELIN TO MONITOR MALIGNANT PLEURAL MESOTHELIOMA}

DT Arnold, D De Fonseka, L Stadon, A Morley, E Keenan, M Darby, L Armstrong, P Virgo, NA Maskell. Academic Respiratory Unit, School of Clinical Sciences, University of Bristol, Bristol, UK

\subsection{6/thoraxjnl-2017-210983.27}

Background Radiological monitoring of malignant pleural mesothelioma (MPM) using modified RECIST criteria is limited by low sensitivity and inter-observer variability. Serial serum mesothelin measurement has shown utility in the assessment of treatment response during chemotherapy but has never been assessed in the longer term follow up of patients.

Methods This is a single centre study of consecutive patients diagnosed with MPM who received chemotherapy or best supportive care (BSC). Serum mesothelin measurements with paired 6 monthly CT scans were performed following the completion of chemotherapy, or from baseline in the BSC group. Changes in mesothelin were correlated with radiological progression and overall survival.

Results Forty-one patients with MPM were recruited and followed up for a minimum of 12 months (range 12-21 months). The majority of patients $(n=23)$ received chemotherapy with pemetrexed and cisplatin. Across the cohort a 10\% rise in serum mesothelin could predict radiological progression with a sensitivity of $96 \%$ (IQR; 79-100) and specificity of 74\% (IQR; 50-91) (figure 1). Sensitivity fell to $80 \%$ in sarcomatoid only disease. Patients with a rising mesothelin at 6 months had significantly worse overall survival (175 days) compared to stable/falling levels (448 days) $(p=0.003)$.

Conclusions This is the first study to assess serum mesothelin's ability to detect progression of MPM following chemotherapy or during BSC. A $10 \%$ rise in serum mesothelin level showed excellent sensitivity at predicting progressive disease. Mesothelin measurement has several advantages over serial CT imaging including reducing hospital visits and cost.

\section{S22 BAP1 EXPRESSION AND TREATMENT OUTCOMES IN MALIGNANT PLEURAL MESOTHELIOMA IN A PROSPECTIVE UK BASED CLINICAL TRIAL}

${ }^{1} \mathrm{~N}$ Kumar, ${ }^{1} \mathrm{~K}$ Kolluri, ${ }^{1} \mathrm{D}$ Al Rifai, ${ }^{1} \mathrm{Y}$ Ishii, ${ }^{2} \mathrm{E}$ Borg, ${ }^{2} \mathrm{M}$ Falzon, ${ }^{3} \mathrm{~A}$ Nicholson, ${ }^{1} \mathrm{~S}$ Janes. ${ }^{1}$ University College London, London, UK; ${ }^{2}$ University College London Hospital, London, UK; ${ }^{3}$ National Heart and Lung Institute, Imperial College, London, UK

\subsection{6/thoraxjnl-2017-210983.28}

Objectives Genomic studies of malignant pleural mesothelioma (MPM) have identified frequent mutations in the nuclear deubiquitinase BRCA Associated Protein 1 (BAP1). Previous studies have identified $100 \%$ correlation between BAP1 nuclear staining and wild type BAP1 status, pointing to immunohistochemistry (IHC) as a reliable technique to detect BAP1 molecular status. The objective of this study is to assess BAP1 expression and infer molecular status using IHC in a cohort from a prospective UK based clinical trial (MSO1). Furthermore, we aim to evaluate the effect of BAP1 status on treatment outcomes.

Methods BAP1 expression was evaluated by IHC in 79 biopsies independently by two consultant histopathologists. Cases were considered positive (wild type BAP1) if strong nuclear staining was present and negative (mutant BAP1) if absent.

Results Assessment of BAP1 expression was concordant in 77 of 79 cases (97\%). BAP1 expression was negative in 66 of these 77 cases (86\%). Patient characteristics and the effect of BAP1 expression on treatment outcomes are in Table 1.

Conclusions BAP1 expression was negative in $86 \%$ of MPM tumours suggesting a high frequency of BAP1 mutations in this UK cohort. No significant differences in clinical characteristics or outcomes were noted between cases with positive or negative BAP1 expression overall. When analysed by treatment subgroup, there was a trend towards a survival benefit in cases with negative BAP1 expression (BAP1 mutants) in the ASC plus vinorelbine arm, but no statistically significant difference in outcomes within any treatment arm. We plan to further validate our findings by correlating BAP1 expression directly with BAP1 molecular status in this cohort using laser capture microdissection and sequencing.

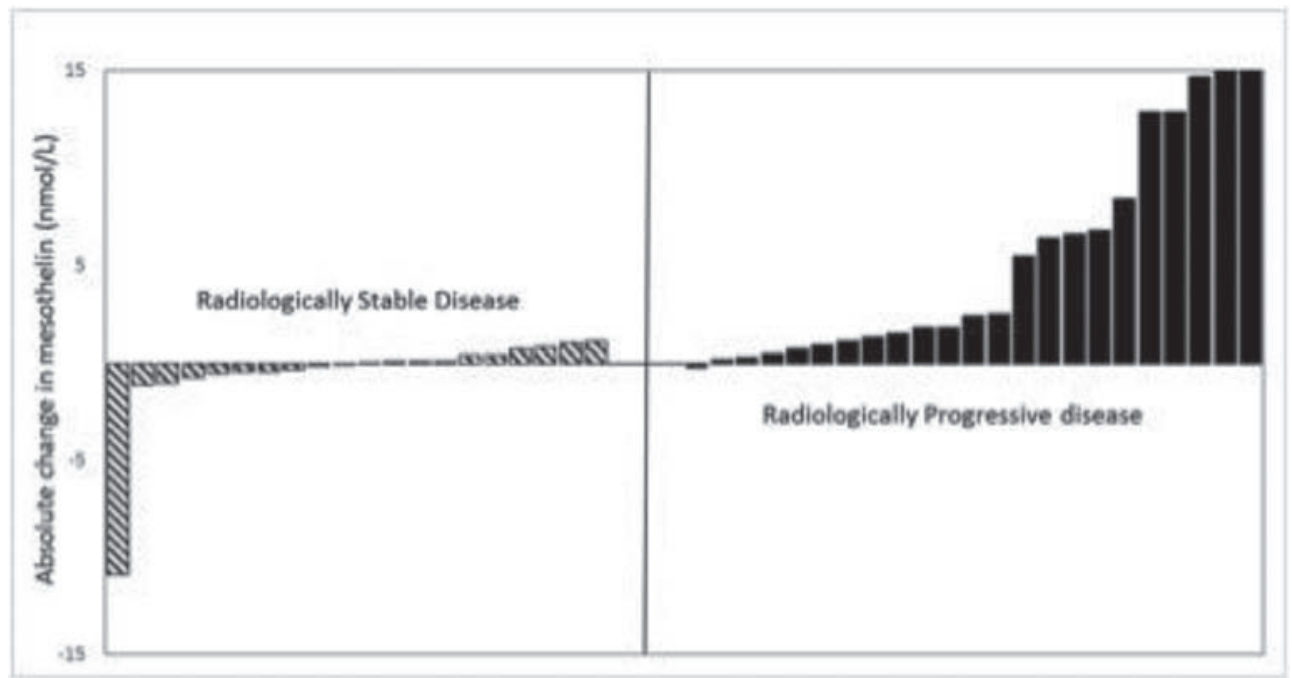

Abstract S21 Figure 1 